\title{
Discovery of new susceptibility genes: proceed cautiously
}

\author{
Tobias Else, $\mathrm{MD}^{1}$ and Lauren Fishbein, $\mathrm{MD}, \mathrm{PhD}^{2}$ \\ Genetics in Medicine (2018) 20:1512-1514; https://doi.org/10.1038/s41436-018-0139-9
}

Pheochromocytoma and paraganglioma (PCC/PGL) are solid tumors in which to date $35-40 \%$ are associated with germline disease-causing variants in one of many described susceptibility genes. ${ }^{1}$ The more common susceptibility genes include the classic tumor suppressors NF1,VHL, and RET, as well as some of the Succinate Dehydrogenase subunit genes ( $S D H B$, $S D H C, S D H D)$. Less commonly, germline pathogenic variants are found in SDHA, TMEM127, MAX, SDHAF2, and FH (the latter two based on case series), and somatic mosaic variants in EPAS1 have been associated with PCC/PGL. Following guidelines from the American Society of Clinical Oncologists ${ }^{2}$ and the American College of Medical Genetics and Genomics (ACMG), ${ }^{3}$ experts recommend that all patients with PCC/ PGL be referred for clinical genetic testing, and if positive, cascade genetic testing should be offered to blood relatives. This testing is now almost uniquely performed through targeted next-generation sequencing (NGS) panels. In this issue, two studies, one by Calsina et al. ${ }^{4}$ and one by Remacha et al., ${ }^{5}$ describe evidence for new potential PCC/PGL susceptibility genes, $M D H 2$ and $D N M T 3 A$, respectively. These intriguing and exciting data also exemplify the need for caution and careful language when describing potential new disease susceptibility genes.

Clinically, identifying a patient with a pathogenic variant in a susceptibility gene leads to tailored surveillance and screening recommendations, which can be costly, timeintensive, risk-bearing, and anxiety-producing. Therefore, the utmost diligence is needed to determine the true risk associated with a certain gene variant to justify the not inconsequential impact on health-care costs and patients' lives. Two prerequisites are needed to understand a variant's significance in causing an increased risk of disease. First, loss or gain of the protein function must be proven to be associated with the disease phenotype, and second, the individual variants observed within the gene need to be associated with disease risk.

For PCC/PGL susceptibility genes, there is a range of associated risk of developing disease phenotype. The initial cancer syndromes predisposing to PCC/PGL, neurofibromatosis type 1, von Hippel-Lindau disease, and multiple endocrine neoplasia type 2, are all high penetrance disorders, meaning most people with a pathogenic variant in the associated gene will develop disease, albeit with variable expressivity for the many manifestations of each syndrome. The underlying genes for these syndromes (NF1, VHL, and $R E T$, respectively) were defined by positional cloning and linkage analysis of germline DNA from large pedigrees. Subsequent genetic testing confirmed the presence of variants in these genes in other large cohorts with disease, and the genetic basis and penetrance became well-established.

Over the past few decades, several other genes that increase the risk for PCC/PGL have been described using similar methodology. $S D H B, S D H C$, and $S D H D$ were established as germline susceptibility genes for hereditary paraganglioma-pheochromocytoma syndromes. Although initially believed to be associated with high penetrance of PCC/PGL, the current estimates are much lower (especially for $S D H B$ and $S D H C$ ).

There are additional PCC/PGL susceptibility genes for which varying amounts of evidence exist to support potential pathogenicity. Although they are included in several clinical targeted NGS panels, they are not all proven to be pathogenic for PCC/PGL beyond a reasonable doubt. Examples of this include variants in SDHAF2 and $F H$. Only a handful of families with SDHAF2 or FH variants are reported with PGL, ${ }^{1}$ barely reaching a confident level of support for true involvement in development of PGL. Also, penetrance must be considered when discussing susceptibility genes. For example, a reasonable amount of evidence suggests that variants in SDHA are associated with an increased risk of PCC/PGL; however, penetrance appears to be extremely low. ${ }^{6}$ Taken together, physicians and genetic counselors who order clinical PCC/PGL genetic testing panels need to be cautious when interpreting the results of variants in these genes and when recommending follow up for patients.

Once a gene is proven to be a susceptibility gene, in that loss or gain of protein function is definitively associated with an increased risk of disease, the next step is to determine if a

\footnotetext{
${ }^{1}$ Division of Metabolism, Endocrinology, \& Diabetes, Department of Internal Medicine, University of Michigan Health System, Ann Arbor, Michigan, USA; ${ }^{2}$ Division of Endocrinology, Metabolism and Diabetes and the Division of Biomedical Informatics and Personalized Medicine, Department of Medicine, University of Colorado School of Medicine, Aurora, Colorado, USA. Correspondence: Lauren Fishbein (lauren.fishbein@ucdenver.edu)
} 
specific variant in the gene is truly pathogenic for the disease. A pathogenic variant should be rare in the general population with a frequency at least less than $1 \%$ in large databases such as ExAC. ${ }^{7}$ Before large databases were publicly available (with the exception of certain founder disease-causing variants), variants were assumed to be pathogenic if found in a biologically plausible gene in patients with disease, even without checking a control population, or if that variant was more frequently found in cases than controls. However, now many of these variants have been reclassified as simply less common variants or variants associated with non-Caucasian populations. Two recent studies exemplify this point. Walsh et $\mathrm{al}^{8}{ }^{8}$ used the ExAC database to compare with a cohort of over 7800 patients with cardiomyopathy and found that some genes in which variants were previously believed to be pathogenic were in fact low frequency normal variants. Newey et al. ${ }^{9}$ showed that across 15 PCC/PGL susceptibility genes commonly tested on clinical targeted NGS panels, even rare variants with allele frequencies less than $0.05 \%$ were observed in 1 in 12 subjects who are presumably disease-free in ExAC.

Pathogenic variants should disrupt protein function (gain or loss). In silico prediction tools are helpful to predict if a missense variant will influence the amino acid sequence or impact protein function, but many rely on evolutionary conservation, which can be misleading depending on the protein function..$^{10}$ Furthermore, it is important to note that there are complexities in interpretation of even variants considered pathogenic. For example, splice site variants may lead to still functional proteins depending on tissue and cell context, and variants encoding a premature stop in translation, especially those in the terminal exon of gene, may escape nonsense-mediated decay and lead to truncated proteins that are retained in the cell. Consequently, the context of the genetic variant must be taken into consideration and functional analysis is critical to determine variant pathogenicity. The ACMG has published guidelines for the interpretation of germline sequence variants, and suggest functional evidence is paramount to determining pathogenicity. ${ }^{11}$

Despite these concerns, it remains crucial that research for new genes associated with disease predisposition continues. The studies in this issue by Calsina et al. ${ }^{4}$ and Remacha et al. ${ }^{5}$ show data in support for two new potential PCC/PGL susceptibility genes, $M D H 2$ and $D N M T 3 A$, respectively. Both groups used the approach of massively parallel sequencing for detecting possible susceptibility genes by sequencing individual patients or clusters of patients with PCC/PGL to find potentially disease-causing genes either by identifying overlapping genes with damaging variants shared between patients with disease or by identifying de novo variants between the proband and their parents without disease. $M D H 2$ was previously reported as a potential susceptibility gene by the same group. ${ }^{12}$ In the prior study, a germline $\mathrm{MDH} 2$ splice site variant was found in one patient with metastatic PCC/PGL leading to alternative splicing, a build-up of malate and the absence of MDH2 protein. ${ }^{12}$ Calsina et al. ${ }^{4}$ now provide data on four additional potentially pathogenic or likely pathogenic germline $\mathrm{MDH} 2$ variants by showing impaired enzymatic function, low $R B P 1$ expression (a marker of Krebs cycle disruption), and/or loss of heterozygosity (LOH). DNMT3A has not previously been described as a potential PCC/PGL susceptibility gene. Remacha et al. ${ }^{5}$ describe two patients with PCC/PGL with possible de novo gain-of-function variants. The authors show that the variants in DNMT3A lead to altered methylation profiles and differential histone $\mathrm{H} 3$ methylation.

These two studies add invaluable data to a growing list of potential risk-associated genes for PCC/PGL. In interpreting these findings, especially for clinical use, it is, however, important to use careful language. Although these studies provide strong evidence that these two genes might be involved in PCC/PGL predisposition, further research is needed to truly classify them as pathogenic genes. Hence, any variant change in these genes, even the ones clearly disrupting function, needs to correctly be referred to as potentially pathogenic, as one cannot apply the ACMG criteria to genes that are not proven beyond a reasonable doubt to be causative for disease predisposition.

The data from both the Calsina et al. ${ }^{4}$ and Remacha et al. ${ }^{5}$ studies are exciting for the field, and set a solid basis for future research on $M D H 2$ and DNMT3A as PCC/PGL susceptibility genes, including testing of large populations of patients with PCC/PGL to determine the contribution of these genes to PCC/PGL predisposition. Any new PCC/PGL susceptibility gene at this point is likely to be associated with low penetrance. Low penetrance genes and/or low risk alleles pose an additional clinical challenge of uncertainty regarding when to justify regular screening and surveillance, particularly taking into account the associated negative effects on patient anxiety and health-care costs.

Costs for targeted NGS panels have significantly decreased and are minimally altered by increasing the number of genes from 10 to 20, for example (indeed most companies will sequence more genes, but only report the requested genes). Therefore, an argument can be made for broad clinical testing of a larger number of proven and suspected genes associated with PCC/PGL, as long as laboratories and clinicians adhere to three rules: (1) all new variants must be added to larger publicly available annotated databases so that information can be gained over time and shared; (2) curation of these data should be centralized to facilitate clinical interpretation; and (3) clinicians must act cautiously on rare variants and only recommend clinical action for truly established pathogenic genes and variants. To this end, the National Institutes of Health (NIH) has funded an online resource called ClinGen (Clinical Genome Resource; https://www.clinicalgenome.org) serving as a central database to help standardize the clinical significance and interpretation of genes and variants for clinical use and research efforts.

\section{ACKNOWLEDGEMENTS}

L.F. was supported by the American Cancer Society (MRSG-15063-01-TBG). 


\section{DISCLOSURE}

The authors declare no conflicts of interest.

\section{REFERENCES}

1. Fishbein L. Pheochromocytoma and paraganglioma: genetics, diagnosis, and treatment. Hematol Oncol Clin North Am. 2016;30:135-150.

2. Robson ME, Storm CD, Weitzel J, et al. American Society of Clinical Oncology policy statement update: genetic and genomic testing for cancer susceptibility. J Clin Oncol. 2010;28:893-901.

3. Hampel $H$, Bennett $R L$, Buchanan $A$, et al. A practice guideline from the American College of Medical Genetics and Genomics and the National Society of Genetic Counselors: referral indications for cancer predisposition assessment. Genet Med. 2015;17:70-87.

4. Calsina B, Currás-Freixes $M$, Buffet $A$, et al. Role of $M D H 2$ pathogenic variant in pheochromocytoma and paraganglioma patients. Genet Med. 2018 Jul 16. https://doi.org/10.1038/s41436-018-0068-7. [Epub ahead of print]

5. Remacha L, Currás-Freixes M, Torres-Ruiz R, et al. Gain-of-function mutations in DNMT3A in patients with paraganglioma. Genet Med. 2018 May 8. https://doi.org/10.1038/s41436-018-0003-y. [Epub ahead of print]
6. Bausch B, Schiavi $\mathrm{F}, \mathrm{Ni}$ Y, et al. Clinical characterization of the pheochromocytoma and paraganglioma susceptibility genes SDHA, TMEM127, MAX, and SDHAF2 for gene-informed prevention. JAMA Oncol. 2017;3:1204-1212.

7. Lek M, Karczewski KJ, Minikel EV, et al. Analysis of protein-coding genetic variation in 60,706 humans. Nature. 2016;536:285-291.

8. Walsh R, Thomson KL, Ware JS, et al. Reassessment of Mendelian gene pathogenicity using 7,855 cardiomyopathy cases and 60,706 reference samples. Genet Med. 2017;19:192-203.

9. Newey PJ, Berg JN, Zhou K, et al. Utility of population-level DNA sequence data in the diagnosis of hereditary endocrine disease. $J$ Endocr Soc. 2017;1:1507-1526.

10. Tang $\mathrm{H}$, Thomas PD. Tools for predicting the functional impact of nonsynonymous genetic variation. Genetics. 2016;203:635-647.

11. Richards S, Aziz N, Bale S, et al. Standards and guidelines for the interpretation of sequence variants: a joint consensus recommendation of the American College of Medical Genetics and Genomics and the Association for Molecular Pathology. Genet Med. 2015;17: 405-424.

12. Cascon A, Comino-Mendez I, Curras-Freixes M, et al. Whole-exome sequencing identifies MDH2 as a new familial paraganglioma gene. J Natl Cancer Inst. 2015;107:djv053. 